\title{
The Surface Morphology Evolution of GaN Nucleation Layer during Annealing and Its Influence on the Crystal Quality of GaN Films
}

\author{
Lin Shang ${ }^{1} \mathbb{D}^{\mathbb{D}}$, Bingshe $\mathrm{Xu}{ }^{1,2, *}$, Shufang Ma ${ }^{1}$, Qingming Liu ${ }^{1}$, Huican Ouyang ${ }^{1}$, Hengsheng Shan ${ }^{1}$, \\ Xiaodong Hao ${ }^{1}$ and Bin Han ${ }^{1}$
}

1 Institute of Atomic and Molecular Science, Shaanxi University of Science and Technology, Xi'an 710021, China; shanglin@sust.edu.cn (L.S.); mashufang@sust.edu.cn (S.M.);

liuqingming1990@sina.com (Q.L.); oyhc223@163.com (H.O.); hsshan@sust.edu.cn (H.S.); hao.xiaodong@sust.edu.cn (X.H.); binhan@sust.edu.cn (B.H.)

2 Key Laboratory of Interface Science and Engineering in Advanced Materials, Ministry of Education, Taiyuan University of Technology, Taiyuan 030024, China

* Correspondence: xubs@tyut.edu.cn; Tel.: +86-0351-6010311

Citation: Shang, L.; Xu, B.; Ma, S.; Liu, Q.; Ouyang, H.; Shan, H.; Hao, X.; Han, B. The Surface Morphology Evolution of GaN Nucleation Layer during Annealing and Its Influence on the Crystal Quality of GaN Films. Coatings 2021, 11, 188. https:// doi.org/10.3390/coatings11020188

\section{Academic Editor:}

Armando Yáñez-Casal

Received: 12 January 2021

Accepted: 2 February 2021

Published: 5 February 202

Publisher's Note: MDPI stays neutral with regard to jurisdictional claims in published maps and institutional affiliations.

Copyright: (C) 2021 by the authors Licensee MDPI, Basel, Switzerland. This article is an open access article distributed under the terms and conditions of the Creative Commons Attribution (CC BY) license (https:/ / creativecommons.org/licenses/by/ $4.0 /)$.

\begin{abstract}
The surface morphology evolution of GaN nucleation layer (NL) after different annealing time has been investigated by atomic force microscope. The surface morphologies of GaN NL after different annealing time are island-like. It is observed that for 0-min annealing time sample nucleation islands (NIs) are high density and small in size which results in high dislocation density GaN films, while the samples with longer annealing time have low density and large size NIs which results in low dislocation density GaN films. The crystal structure of GaN NLs after different annealing time investigated by high-resolution X-ray diffraction indicates GaN NL is polycrystalline. During annealing, GaN nanocrystals misaligned with sapphire gradually disappear and GaN nanocrystals aligned with sapphire survive and grow up. Thus, the GaN NL surface has different NIs' densities and sizes after different annealing time. The NL annealing time can effectively control the density and size of the NIs and further determine the GaN films crystal quality.
\end{abstract}

Keywords: GaN; annealing time; nucleation island; dislocation

\section{Introduction}

The third-generation semiconductor GaN film materials have been widely used in solid-state lighting, semiconductor blue-green laser, transistor and microwave devices since a high-quality GaN film was successfully grown on sapphire substrate [1-3]. GaN grown on sapphire involves a nucleation layer (NL) grown at low temperature and the growth of a thick GaN grown at high temperature (HT) which is called two-step growth [4]. The role of GaN NL during annealing is to provide nucleation islands (NIs) for the GaN crystallites and to promote the lateral growth of the film. The thermal annealing process was found to have an important effect on the properties of the subsequent GaN film [5]. After the anealing of NL, the GaN NL will transform to three-dimensional (3D) NIs. Threading dislocations (TDs) are usually generated at the boundaries of NIs and inside the NIs. Thus, low density NIs are beneficial to reduce the TD density in GaN film during the lateral growth of 3D islands. TDs are known to have detrimental effects on device performance, such as non-radiative recombination centers, and reduce the lifetime of devices by acting as diffusion channels between the electrodes. Thus, high performance GaN coatings with low dislocation density are used as high-efficiency light emitting diodes and laser diodes. Flat surface morphology of GaN film is good at obtaining multiple quantum well structures with abrupt interfaces. During my experiments, the NIs size and density have a strong relationship with annealing time, so it is necessary to investigate the 3D NIs evolution under different annealing time. Although it has been previously reported that high quality 
GaN films can be obtained by optimizing NL growth parameters, such as nucleation temperature, nucleation time, pressure and trimenthyl gallium (TMGa) and $\mathrm{NH}_{3}$ mole ratio [6-10], there are still few studies on the dislocation evolution mechanism related to the crystal quality of GaN epitaxial layer in terms of NL annealing time.

In this study, the properties of GaN crystals at each stage of the two-step growth process were characterized and the physical mechanism of annealing time on GaN film crystal quality was systematically analyzed. The NIs' size and density after annealing are affected greatly by NL annealing time. The surface morphology of the NLs after different annealing time measured by atomic force microscope (AFM) shows that increasing the NL annealing time causes larger island size and space and further increases the NL annealing time, and the island size decreases instead. Based on crystal growth theory, low density NIs are beneficial to bulk GaN crystal quality and the NI's size and density are closely related with the NL annealing time.

\section{Materials and Methods}

The GaN films with different NL annealing time were grown on c-plane sapphire substrate by TS300 metal organic chemical vapor deposition (MOCVD) system (Aixtron, Herzogenrath, Germany). First, a low-temperature GaN NL of about $25 \mathrm{~nm}$ was deposited on the sapphire at $530{ }^{\circ} \mathrm{C}$. Then the source of TMGa was turned off, the substrate was heated to $1080^{\circ} \mathrm{C}$ and maintained at this temperature for 0,2 and 4 min for annealing treatment. The process of temperature ramps and maintains is known as a recrystallization process and we called the maintenance time at high temperature NL annealing time. Finally, TMGa flow was switch on and a 2- $\mu$ m undoped GaN film was deposited with a growth rate $2 \mu \mathrm{m} / \mathrm{h}$.

A semiconductor laser operating at a wavelength of $632.8 \mathrm{~nm}$ was used as in-situ monitoring tool to investigate the reflectance throughout the growth progress. The surface morphologies were studied by SPA-300HV atomic force microscope (AFM) (Seiko, Tokyo Prefecture, Japan) at tapping model. The full width at half maximum (FWHM) of Highresolution X-ray diffraction (HRXRD Bruker D8 DISCOVER, Karlsruhe, Germany) $\omega$-scan rocking curve was conducted to analyze the crystal properties of GaN epilayers.

\section{Results and Discussion}

The reflectivity traces recorded during the whole growth process of the three samples are shown in Figure 1. The whole growth process is divided into four steps. For the first growth step (step 1), which is the deposition of the NL, the reflectivity increases as GaN has a higher refractive index than sapphire. The increased reflectivity intensity that can provide the thickness of $\mathrm{NL}$ is about $25 \mathrm{~nm}$. When the temperature ramps up to $900{ }^{\circ} \mathrm{C}$, the $\mathrm{GaN} N \mathrm{~L}$ begin to decompose and recrystallize. The surface roughness drops rapidly which results in the reflectivity intensity dropping abruptly, close to zero. With the annealing time increases, the GaN NL not covered with NIs is completely etched away, and the flat sapphire surface increases the reflectivity intensity [11-13]. The stage 3 corresponding to the early stage of un-doped GaN growth at $1080{ }^{\circ} \mathrm{C}$ is the three-dimensional (3D) NI growth. It is obvious that the more annealing time remains, the longer the 3D growth time is. At stage 4, a smooth GaN surface has been established and GaN grows in step flow mode. At this point, the reflectivity begins to oscillate due to interference between flat $\mathrm{GaN}$ surface and $\mathrm{GaN} /$ sapphire interface. At this stage, for a 0-min annealing time sample, the amplitude of oscillation gradually decreases as growth time increases. The amplitude of oscillation has a strong relationship with GaN surface roughness. The larger the amplitude is, the lower the roughness is. Thus, this indicates the film surface roughness of the 0-min annealing time sample gradually increases [14]. 


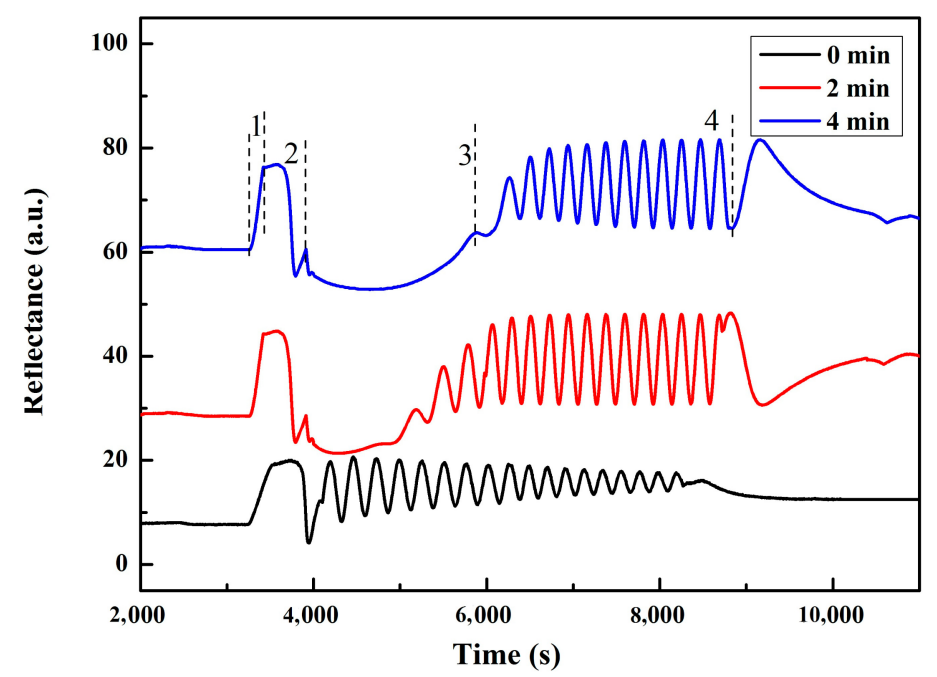

Figure 1. The reflectance recorded during the whole growth process of 0,2 and 4 min annealing time samples.

In order to further explore the influence of annealing time on the GaN film crystal qualities as well as the NIs coalescence time, the GaN NLs after different annealing time were characterized by AFM. The annealing process transforms NL into isolated NIs, as seen in Figure 2. The NIs of zero min annealing time sample have the highest density and the smallest size. As for the two min annealing time sample, the NIs' size becomes bigger and the density decreases obviously. For the four min annealing time sample, the NIs' size becomes bigger and the density decreases further. The zero min annealing time sample forms smaller and high density NIs, which is attributed to NIs' quick coalescence. Thus, for the zero min annealing time sample, reflectivity oscillates rapidly when high temperature $\mathrm{GaN}$ grows. For the four min annealing time sample, larger spaces between NIs cause longer coalescence time.
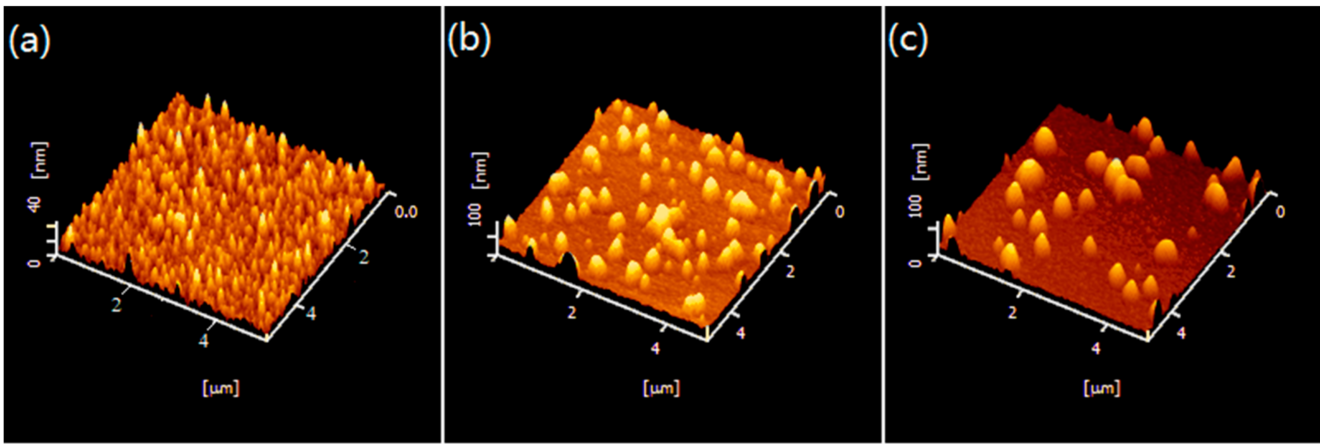

Figure 2. The 3D AFM images $(5 \mu \mathrm{m} \times 5 \mu \mathrm{m})$ for GaN NL after annealing $(\mathbf{a}-\mathbf{c})$ with 0,2 and 4-min annealing time.

Figure 3 shows $5 \mu \mathrm{m} \times 5 \mu \mathrm{m}$ AFM images of bulk GaN layer. As seen in Figure 3, the samples with two and four min annealing time exhibit a smooth surface morphology with easily identified atomic steps and the root mean square (RMS) surface roughness is 0.31 and $0.35 \mathrm{~nm}$, respectively. However, as can be seen for the 0 min annealing time sample, the surface morphology of step kinks is observed as marked by black box, the terraces width is not uniform compared with others. Its RMS surface roughness is $1.21 \mathrm{~nm}$, much larger than others. The step kinks and large RMS roughness may come from the high surface stress induced by high dislocation density [15]. As seen in these figures, the samples with low density and large space NIs exhibit a smooth surface morphology with easily identified atomic steps and terraces compared to the sample with high density and small space NIs. 

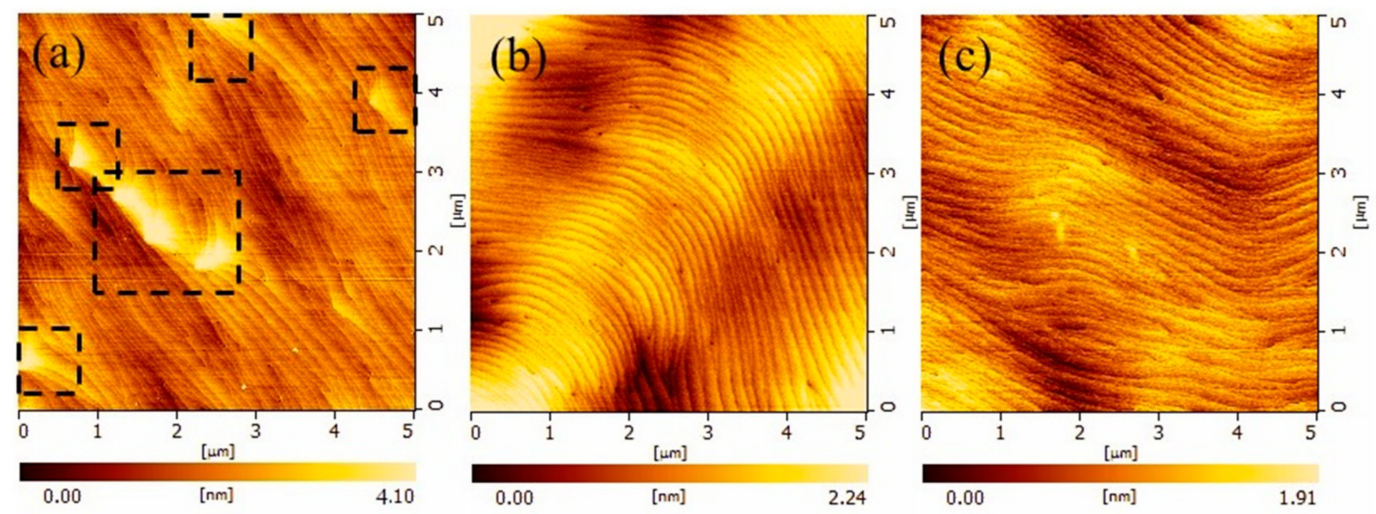

Figure 3. The AFM images ( $5 \mu \mathrm{m} \times 5 \mu \mathrm{m}$ ) of bulk GaN with (a) 0 , (b) 2 and (c) 4-min annealing time. The black boxes in (a) are step kinks.

Annealing is a crucial step in GaN two-step growth progress [16]. Figure 4 shows the HRXRD rocking curves of (002) and (102) planes of bulk GaN and the insert corresponds to their FWHMs. When the annealing time of NL increases from zero to two min, the values of (002) and (102) plane FWHM significantly decreases from 341 to 275 and 448 to 267 arcsec, respectively. With a further increase of annealing time to four min, the FWHM of (002) plane further decreases to 180 arcsec but (102) plane increases to 284 arcsec, instead. The symmetric (002) plane FWHMs show the information about the screw dislocation density that is mainly generated inside NIs $[17,18]$. Asymmetric (102) plane FWHMs show the information about the mixed and pure edge dislocation density that are mainly generated from the coalescence boundary of NIs $[19,20]$. The HRXRD results indicate NI's size and density have important influence on GaN film crystal quality and are influenced by annealing time.

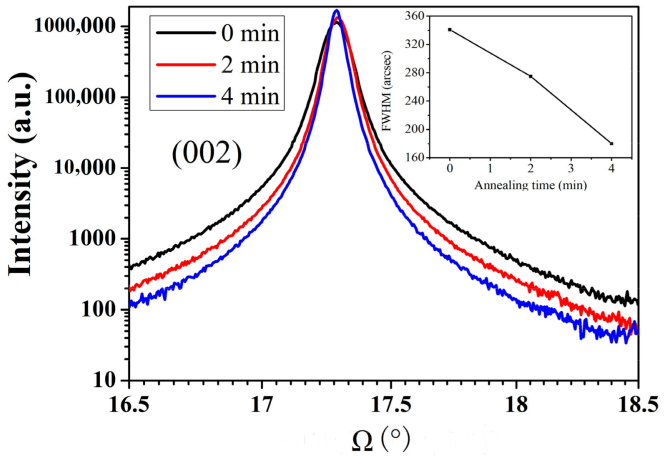

(a)

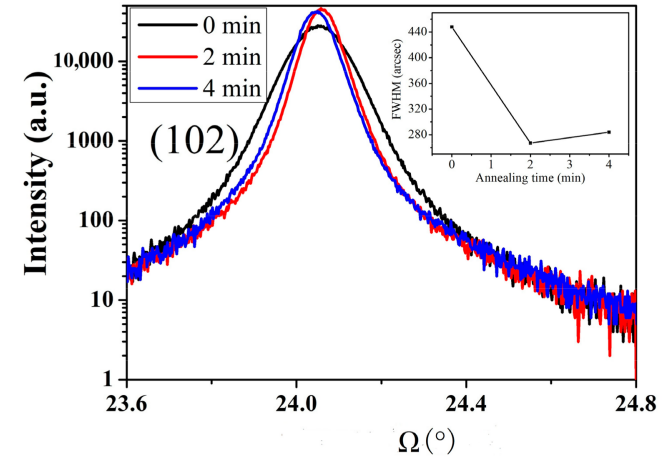

(b)

Figure 4. The rocking curves of (a) (002) and (b) (102) planes of bulk GaN with 0, 2 and 4 min annealing time and the insert corresponds to their FWHMs.

From our experimental results, the NI's size and density are very important for GaN film crystal quality and are closely related with the NL annealing time. In order to analyze the structure evolution during annealing, the GaN NLs before and after different annealing time are characterized by HRXRD (002) plane rocking curve scan, as shown in Figure 5. The (002) rocking curve FWHMs of GaN NL before and after zero, two and four min annealing are 7640, 3474, 1587 and 1302 arcsec, respectively. The FWHM of GaN NL before annealing is very large compared with GaN NL after annealing. Furthermore, the morphology of GaN NL before annealing is measured via AFM, as shown in Figure 6. Before annealing, the surface is covered with dense grains which indicates the NL is consist with mosaic blocks. A mosaic crystal is an idealized model of an imperfect crystal, imagined to consist of numerous small perfect crystals (crystallites) that are to some extent 
randomly misoriented. Thus, GaN NL crystal structure model is raised as shown in Figure 7. This indicates GaN NL is polycrystalline and some crystals are perfect coherent with sapphire substrate and some are to some extent randomly misoriented. The NL annealing is a decomposition-redeposition process proposed by Lorenz [21] and Narayanan [22]. It has been reported that low temperature GaN NL is highly defective with nanocrystals aligned and misaligned with sapphire and nanocrystals appearing amorphous [23]. After annealing, the FWHM of (002) rocking curve decreases obviously which indicates the misoriented GaN crystals decompose during annealing. The FWHM of the two min annealing sample decreases further which indicates that at zero min the misoriented GaN crystals do not decompose completely. The FWHM of the four min sample does not decrease anymore but the diffraction peak intensity decreases obviously compared with two min sample, which indicates that after two min annealing the misoriented GaN crystals decomposed completely, and during annealing between two and four min some coherent $\mathrm{GaN}$ crystals decompose. The results indicate $\mathrm{GaN}$ nanocrystals misaligned with sapphire gradually disappear and the GaN nanocrystals aligned with sapphire survive and grow up during annealing.

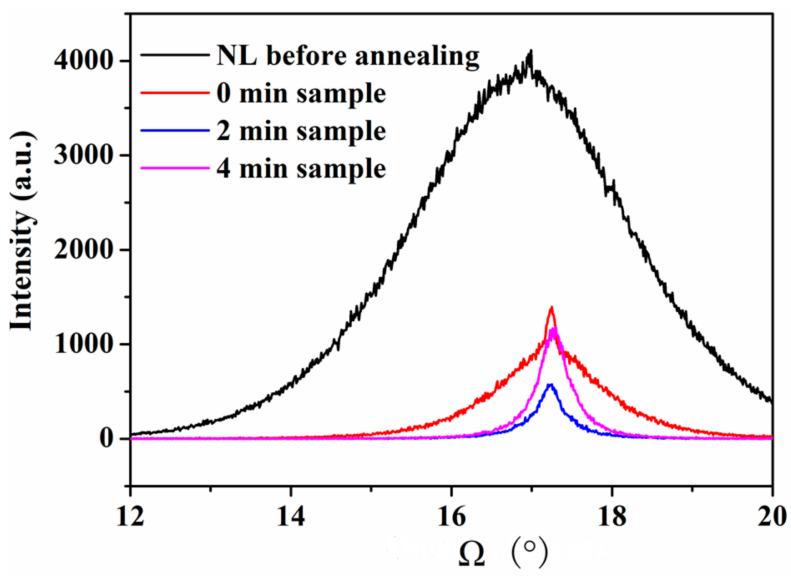

Figure 5. The rocking curves of (002) plane of GaN NL before and after 0, 2 and 4-min annealing.

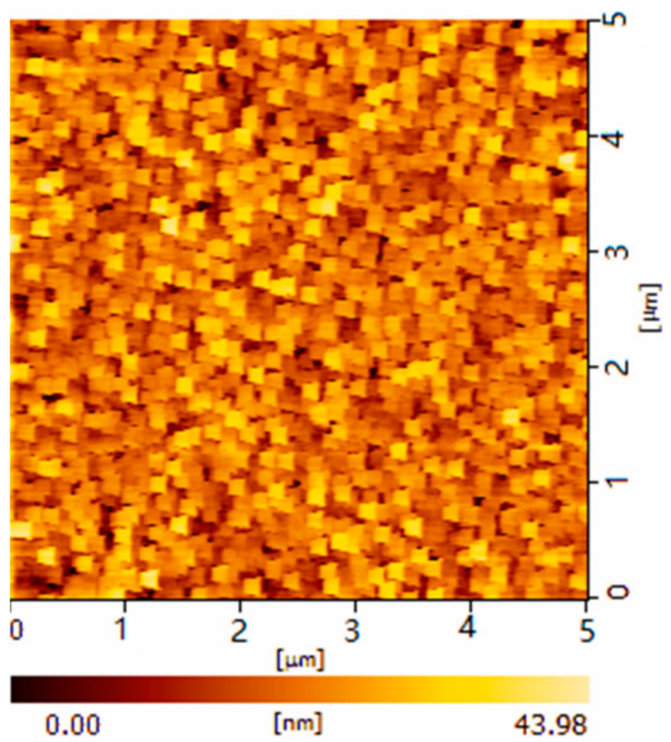

Figure 6. The AFM image of GaN NL before annealing. 


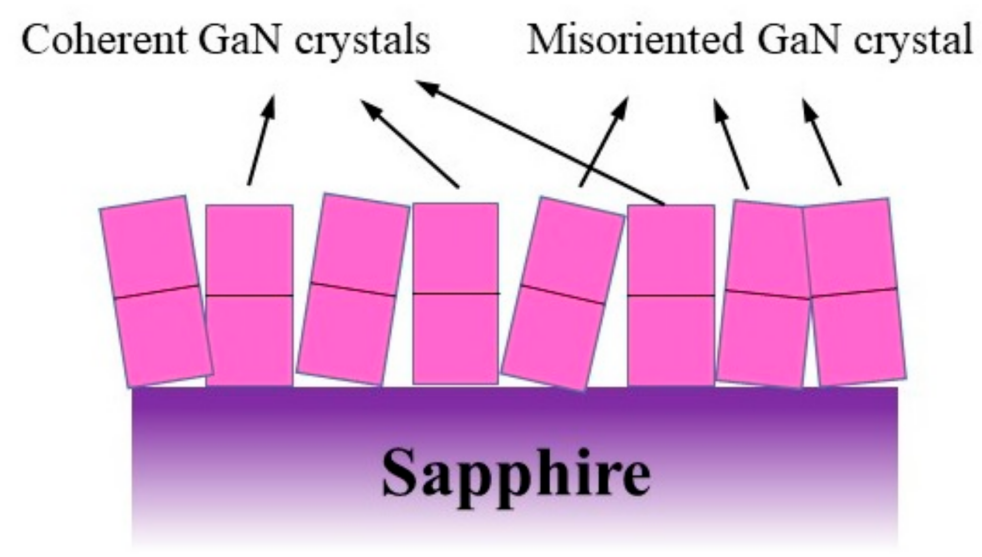

Figure 7. The GaN NL crystal structure model.

In order to illustrate the structure evolution during the annealing stage, a schematic of the structure evolution and associated dislocation generation and propagation processes discussed above is proposed, as shown in Figure 8. GaN nanocrystals aligned with sapphire are stable state and not easy to decompose at high temperature. GaN nanocrystals misaligned with sapphire are in a metastable state. GaN amorphous nanocrystals are in an unstable state and easy to decompose. At the beginning stage of GaN NL annealing, amorphous GaN nanocrystals preferentially decompose. GaN nanocrystals aligned and misaligned with sapphire survive, like Figure $8 \mathrm{~b}$. At the same time $\mathrm{GaN}$ nanocrystals decompose, redepositions occur on GaN nanocrystals re-exposed to the sapphire surface. Thus, after a short annealing time, survived nanocrystals aligned and misaligned with sapphire cause high density GaN islands with high density and small size like Figure 2a. Any misalignment from the perfect epitaxial relationship will introduce larger stress in the $\mathrm{GaN}$ nanocrystals. The larger the stress GaN nanocrystals suffer is, the easier the decomposition of these nanocrystals. As a result, regrowth on misaligned $\mathrm{GaN}$ nanocrystals may be slower than regrowth on the well-aligned nanocrystals. As annealing time continues, amorphous GaN nanocrystals decompose completely and well-aligned islands may keep growing at the expense of misaligned ones, which supposedly decompose easier than the well-aligned ones. Finally, only well-aligned islands survive like Figure 8d. Annealing longer than that will result in a slow diminishing of the GaN islands, since decomposition dominates over redeposition without a new Ga supply. Thus, the size and density of GaN islands depend closely on nucleation time.

During the 3D growth stage, the screw-type dislocations inside NIs first bend and then meet with each other at the coalescence boundary [24]. Dislocations can be annihilated by coalescence with the opposite component of Burgers vector [25], like Figure 8e. Now we can answer the question of why the quality of bulk GaN is sensitive to the NL annealing time. For the zero min annealing time sample, the spaces between the NIs are small, which results in screw-type dislocations that have less chance to bend and terminate. Meanwhile, many edge-type dislocations form at the NIs' boundary due to high density of NIs. As the space between NIs increases, a longer coalescence time for NIs allows dislocations to have more chance to bend and annihilate in pairs. Thus, the sample with low-density GaN NIs is beneficial for the dislocation annihilation because it has lesser coalescence boundaries and longer coalescence space. Thus, for the two min annealing time sample, the screw- and edge-type dislocation density decreases obviously compared with the zero min sample. But for the four min sample, the space between the NIs is too large, which increases the NIs' misoriented degree; the edge-type dislocation density is somewhat higher than the two min sample. 
(a)

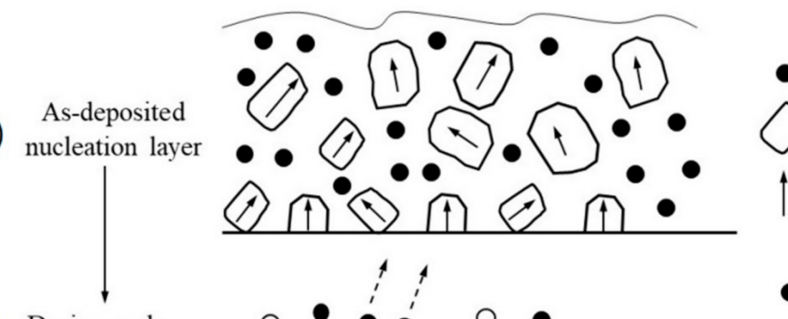

: Amorphous GaN nanocrystals

$\bigcirc$ : GaN nanocrystals

$\uparrow$ : Arrows indicate crystal orientation

(b)

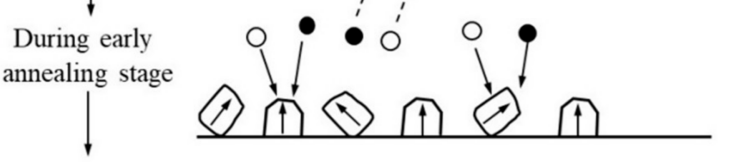

- Ga-containing radicals

$\mathrm{O}: \mathrm{NH}_{3}$-containing radicals

$\longrightarrow$ : redeposition

$\rightarrow$ : to exhuast

(c)

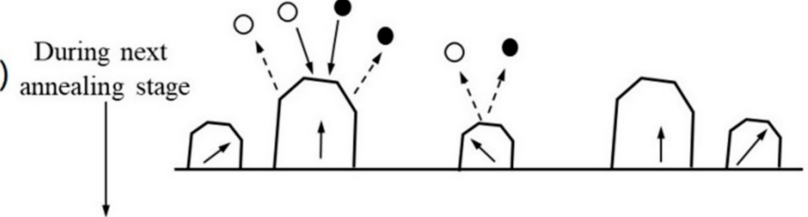

- : Ga-containing radicals

$\mathrm{O}: \mathrm{NH}_{3}$-containing radicals

$\longrightarrow$ : redeposition

(d) After annealing

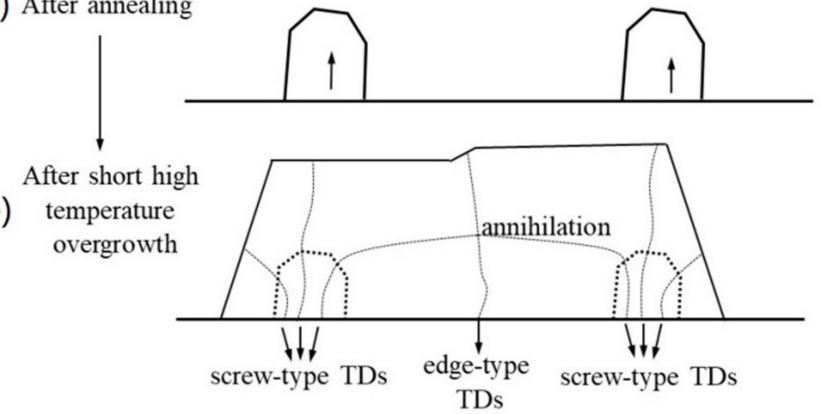

Figure 8. Schematic illustrating structure evolution of GaN nucleation, annealing and 3D growth. (a) is the as-deposited nucleation layer; (b) is during early annealing stage; (c) is during next annealing stage; (d) is after annealing stage; (e) is during high temperature overgrowth stage.

\section{Conclusions}

In summary, the GaN film crystal quality depends on the size and density of NIs that form after different annealing times. The zero min annealing time sample with small- and high-density NIs tends to grow GaN film with numerous edge-type dislocations at the NIs' boundaries. Small NI space is not beneficial for screw-type dislocation bending and annihilation. Thus, the zero min annealing time sample has the highest (002) and (102) plane FWHMs. As for the four min annealing time sample, it has the lowest NI density, which causes the lowest density screw-type dislocation density, thus it has the lowest (002) plane FWHM. However, the space between the NIs is too large, which increases the NIs' misoriented degree. The edge-type dislocation density is somewhat higher than the two min annealing time sample which increases its (102) plane FWHM. The two min annealing time sample has the lowest total dislocation density because it has low-density and uniform NIs.

Author Contributions: Conceptualization, B.X. and S.M.; validation, L.S.; formal analysis, Q.L.; investigation, H.O.; resources, X.H., B.H., and H.S.; data curation, Q.L. and H.O.; writing-original draft preparation, L.S. and S.M.; writing-review and editing, B.X., X.H., B.H., and H.S. All authors have read and agreed to the published version of the manuscript.

Funding: This work is supported by the National Key R\&D Program of China (2016 YFB0401803). The National Natural Science Foundation of China (21972103, 51901119, 21902096). Open Project of Key Laboratory of Interface Science and Engineering in Advanced Materials (KLISEAM201904). Natural Science Special Project of Education Department of Shaanxi Province (No. 19JK0136). Shanxi Key Research and Development Project (201703D111026). The Research Starting Foundation of Shaanxi University of Science and Technology (2018BJ-36, 2017BJ-55, 2017BJ-53). 


\section{Data Availability Statement: Not applicable.}

Conflicts of Interest: The authors declare no conflict of interest.

\section{References}

1. Sivanathan, P.C.; Shuhaimi, A.; Hamza, H.; Kowsz, S.J.; Muhammad, I.M.; Khudus, A.; Li, H.; Allif, K. Effect of thermal interaction between bulk GaN substrates and corral sapphire on blue light emission InGaN/GaN multi-quantum wells by MOCVD. Superlattices Microstruct. 2018, 119, 157-165. [CrossRef]

2. Hu, X.; Xiao, F.; Zhou, Q.; Zheng, Y.; Liu, W. High-luminous efficacy green light-emitting diodes with InGaN/GaN quasisuperlattice interlayer and Al-doped indium tin oxide film. J. Alloys Compd. 2019, 794, 137-143. [CrossRef]

3. Ni, R.; Chuo, C.; Yang, K.; Ai, Y.; Zhang, L.; Cheng, Z.; Liu, Z.; Jia, L.; Zhang, Y. AlGaN-based ultraviolet light-emitting diode on high-temperature annealed sputtered AlN template. J. Alloys Compd. 2019, 794, 8-12. [CrossRef]

4. Shmidt, N.M.; Chernyakov, A.E.; Tal, N.A.; Nikolaev, A.E.; Sakharov, A.V.; Petrov, V.N. The impact of the surface morphology on optical features of the green emitting InGaN/GaN multiple quantum wells. J. Cryst. Growth 2019, 520, 82-84. [CrossRef]

5. Chen, Y.; Chen, Z.; Li, J.; Chen, Y.; Li, C.; Zhan, J.; Yu, T.; Kang, X.; Jiao, F.; Li, S.; et al. A study of GaN nucleation and coalescence in the initial growth stages on nanoscale patterned sapphire substrates via MOCVD. CrystEngComm 2018, 20, 6811-6820. [CrossRef]

6. Yi, L.; Frentrup, M.; Vacek, P.; Massabuau, F.C.; Kappers, M.J.; Wallis, D.J.; Oliver, R.A. Investigation of MOVPE-grown zincblende GaN nucleation layers on 3C-SiC/Si substrates. J. Cryst. Growth 2019, 524, 125-167.

7. Bi, Z.; Gustafsson, A.; Lenrick, F.; Lindgren, D.; Hultin, O.; Wallenberg, L.R.; Jonas, B.; Monemar, B.; Samuelson, L. High in-content InGaN nano-pyramids: Tuning crystal homogeneity by optimized nucleation of GaN seeds. J. Appl. Phys. 2018, 123, 025102. [CrossRef]

8. Su, X.; Ye, T.; Wang, S.; Shi, Y.; Fan, L.; Liu, L.; Zhang, G.; Shi, X.; Wei, M.; Su, X.; et al. Surface morphology of GaN nucleation layer grown by MOCVD with different carrier gas surface morphology of GaN nucleation layer grown by MOCVD with different carrier gas. AIP Adv. 2018, 075301, 1-7.

9. Applicata, O. Optimisation of LT-GaN nucleation layer growth conditions for the improvement of electrical and optical parameters of GaN layers. Opt. Appl. 2019, 49, 167-176.

10. Li, W.; Xu, S.; Zhang, Y.; Peng, R.; Du, J.; Zhao, Y.; Fan, X.; Zhang, J.; Tao, H.; Wang, X.; et al. GaN quality evolution according to carrier gas for the nucleation layer and buffer layer. Opt. Mater. Express 2019, 9, 1945-1954. [CrossRef]

11. Kim, D.; Moon, Y.; Ahn, K.; Park, S.; Kim, D.; Moon, Y.; Ahn, K.; Park, S. In situ normal incidence reflectance study on the effect of growth rate of nucleation layer on GaN by metalorgani. J. Vac. Sci. Technol. B 2000, 140, 140-143. [CrossRef]

12. Lünenbürger, M.; Protzmann, H.; Heuken, M.; Jürgensen, H. In situ monitoring of GaN growth in multiwafer MOVPE reactors. Phys. Status Solidi A 1999, 176, 727-731. [CrossRef]

13. Charles, M.; Mrad, M.; Kanyandekwe, J.; Yon, V. Extraction of stress and dislocation density using in-situ curvature measurements for AlGaN and GaN on silicon growth. J. Cryst. Growth 2019, 517, 64-67. [CrossRef]

14. Von Dollen, P.; Pimputkar, S.; Alreesh, A.; Nakamura, S.; Speck, J.S.; Von Dollen, P.; Pimputkar, S.; Alreesh, M.A.; Nakamura, S. A new system for sodium flux growth of bulk gan part II: In situ investigation of growth processes. J. Cryst. Growth 2016, 456, 67-72. [CrossRef]

15. Barchuk, M.; Röder, C.; Shashev, Y.; Lukin, G.; Motylenko, M.; Kortus, J.; Pätzold, O.; Rafaja, D. Correlation between the residual stress and the density of threading dislocations in GaN layers grown by hydride vapor phase epitaxy. J. Cryst. Growth 2014, 386, 1-8. [CrossRef]

16. Feng, S.; Chen, Y.; Lai, C.; Tu, L.; Han, J. Anisotropic strain relaxation and the resulting degree of polarization by one- and two-step growth in nonpolar a-plane GaN grown on r-sapphire substrate. J. Appl. Phys. 2013, 233103, 1-7. [CrossRef]

17. Chierchia, R.; Böttcher, T.; Heinke, H.; Einfeldt, S.; Figge, S.; Chierchia, R.; Heinke, H.; Einfeldt, S.; Figge, S.; Hommel, D. Microstructure of heteroepitaxial GaN revealed by X-ray diffraction. J. Appl. Phys. 2013, 93, 8918-8925. [CrossRef]

18. Heinke, H.; Kirchner, V.; Einfeldt, S.; Hommel, D. X-ray diffraction analysis of the defect structure in epitaxial GaN. Appl. Phys. Lett. 2000, 77, 2145-2147. [CrossRef]

19. Search, H.; Journals, C.; Contact, A.; Iopscience, M.; Address, I.P. In-plane imperfections in GaN studied by X-ray diffraction. J. Phys. D Appl. Phys. 2005, 38, A99-A104.

20. Kushvaha, S.S.; Kumar, M.S.; Maurya, K.K.; Dalai, M.K.; Sharma, N.D. Highly c-axis oriented growth of GaN film on sapphire (0001) by laser molecular beam epitaxy using HVPE grown GaN bulk target. AIP Adv. 2014, 092109, 1-10. [CrossRef]

21. Lorenz, K.; Gonsalves, M.; Kim, W.; Narayanan, V.; Mahajan, S.; Lorenz, K.; Gonsalves, M.; Kim, W.; Narayanan, V.; Mahajan, S. Comparative study of GaN and AlN nucleation layers and their role in growth of GaN on sapphire by metalorganic. Appl. Phys. Express 2013, 3391, 1-4.

22. Taylor, P.; Narayanan, V. Gallium nitride epitaxy on (0001) sapphire. Philos. Mag. A 2002, 82, 885-912.

23. Meng, F.Y.; Han, I.; Mcfelea, H.; Lindow, E.; Bertram, R.; Werkhoven, C.; Arena, C.; Mahajan, S. Structural evolution of GaN layers grown on (0001) sapphire by hydride vapor phase epitaxy. J. Cryst. Growth 2011, 327, 13-21. [CrossRef]

24. Ning, X.J.; Manufacturing, S.; Corporation, I.; Pirouz, P. Kink and crack interfaces in deformed 6 H-SiC single crystals. MRS Proc. 1994, 357, 157-165. [CrossRef]

25. Weng, X.; Raghavan, S.; Acord, J.D.; Jain, A.; Dickey, E.C.; Redwing, J.M. Evolution of threading dislocations in MOCVD-grown GaN films on (111) Si substrates. J. Cryst. Growth 2007, 300, 217-222. [CrossRef] 